\section{A novel concept of pneumatic pump in the outpatient man- agement of mixed leg ulceration: a pilot study}

\author{
Mirko Tessari, Sergio Gianesini, Paolo \\ Spath, Erica Menegatti, Maria Grazia \\ Sibilla, Matilde Zamboni, Anna Maria \\ Malagoni, Paolo Zamboni \\ Unit of Translational Surgery and \\ Vascular Diseases Center, University \\ Hospital of Ferrara, Italy
}

\begin{abstract}
Mixed leg ulcer is a challenge in vascular diseases because both arterial and venous systems of the lower extremities are involved in the wound pathogenesis. Management is very difficult because the coexistence of the two conditions sometimes prevent to use compression bandaging, which is the cornerstone of venous leg ulcer treatment. It has been recently developed the so called gradient pump (GP), a novel device which permits to intermittently stop the flow in the femoral vein with a pneumatic cuff placed on the thigh. Subsequent release of the femoral vein compression determines a dramatic aspiration of the blood up-ward the heart, with synchronous and measured improved perfusion of the distal limb. We applied GP at 4 consecutive cases of multiple mixed painful ulcerations of the foot and leg scheduled for below knee and/or foot amputations. In all cases measure of pain by visual analogue scale significantly decreased; GP in most cases together intravenous infusion of synthetic prostacyclin led to improvement and/or minimal amputation. Therefore such a management can be done on outpatient bases, allowing a considerable lowering of health costs. We conclude that GP could be a valuable tool in the arsenal of mixed leg ulcer clinical assessment.
\end{abstract}

\section{Introduction}

It has been recently demonstrated that a novel intermittent pneumatic compression (IPC) device based on a new hemodynamic concept respect to standard pneumatic compression for lower limb ischemia, ${ }^{1}$ when administrated with an interval protocol significantly improves the foot perfusion. This result has been measured by the mean of near infrared spectroscopy (NIRS) in ischemic legs of patients affected by peripheral arterial disease (PAD) of the lower limbs. ${ }^{2}$ The same effect was not observed by a traditional sequential foot-calf device after $35 \mathrm{~min}$ or more of treatment. The new IPC original device was conceived to evoke a positive hemodynamic effect not by a bottom up but a top-down action. The proximal inflation at thigh, and the cyclic squeezing at the femoral vein, aims to create a pressure gradient to favor the empty of the distal veins in the decompression phase and the drainage of the congest foot, as well (Figure 1).

According to the analysis of the traces obtained by NIRS ${ }^{2,3}$ recording of patients previously tested, we observed that a therapeutic cycle based on 5 min working and 5 min resting, resulted optimal for the majority of PAD patients, along a total time of $30 \mathrm{~min}$.

Mixed leg ulceration (MLU) is a really challenging medical condition because chronic venous insufficiency, mostly of the superficial veins, is coupled with $\mathrm{PAD}$, often induced by some severe chronic disease such as diabetes, etc. ${ }^{4-8}$ Treatment is sometimes embarrassing especially when considering compression, which is the cornerstone of venous leg ulcer treatment. ${ }^{9,10}$ Inelastic or low grade compression are suggested in these cases,${ }^{6,9}$ but the compliance could be very low on behalf of the patients because of the pain. Pain is the main reason of poor compression compliance as well as of poor quality of life in MLU. ${ }^{11,12}$

One more reason of difficulty in applying compression in MLU is the contemporaneous wide spread of negative pressure devices in managing infections of the wound. ${ }^{13}$ Negative pressure devices aspirate exudates from the wound, especially in the distal part of the leg and foot. ${ }^{14-16}$

While this effect is advantageous for infection treatment it is of course not advisable for improving hemodynamics and perfusion. Therefore, compression increases the external pressure component of the resulting in favorable pressure for drainage and oxygenation. To the contrary, negative pressure of vacuum device decreases the external pressure component of transmural pressure so affecting hemodynamics and perfusion.

We herein report the application of gradient pump (GP) in some cases of MLU, measuring the effect on wound healing and pain control.

\section{Case Series}

\section{Protocol used}

All the subsequent clinical cases underwent to GP treatment for MLU. The $\mathrm{GP}^{2}$ consists of an inflatable sleeve having a length of 50-80 $\mathrm{cm}$ and a width of $13 \mathrm{~cm}$, to be positioned over the mid thigh with a rigid element inside (length: $9 \mathrm{~cm}$, width: $10 \mathrm{~cm}$ ) to selectively improve the compressive effect on the femoral
Correspondence: Mirko Tessari, Vascular Diseases Center, University Hospital of Ferrara, via Aldo Moro 8, 44124 Cona (FE), Italy

Tel.: +39.0532.239498.

E-mail: mirko.tessari@unife.it

Key words: Pneumatic compression; gradient pump; mixed leg ulcer; visual analogue scale; limb perfusion.

Conflict of interest: the authors declare no potential conflict of interest.

Conference presentation: this paper has been presented at the International meeting Veinland, Isola di Albarella Italy, May 18th 2014.

Received for publication: 12 May 2015.

Revision received: 11 April 2016.

Accepted for publication: 11 April 2016.

This work is licensed under a Creative Commons Attribution 4.0 License (by-nc 4.0).

() Copyright M. Tessari et al., 2016

Licensee PAGEPress, Italy

Veins and Lymphatics 2016; 5:5266

doi:10.4081/vl.2016.5266

vein. The inflatable cuff is connected to a compressor that produces variable levels of pressure set by a manual regulator. We set the pressure at $120 \mathrm{mmHg}$, reducing this value only when the systemic arterial pressure was less. Furthermore, the device includes a manual electromechanical timer to produce periodic sequences of pressure and the modification and control of the working/resting cycles. The GP was set to deliver an operative cycle of a progressive inflation with a compression phase lasting $20 \mathrm{~s}$ and a later decompression phase lasting $40 \mathrm{~s}$ (1 cycle/min).

\section{Case \#1}

Patient A is a 57 -year old male affected by MLU due to association of post thrombotic syndrome (previous thrombosis of the femoral vein 10 years before) with distal emboli from popliteal aneurysm (substitution with polytetrafluoroethylene graft). The critical limb ischemia and the severity of venous disease determined ulceration of the foot and fingers, complicated by infection and necrosis. Pain measured by visual analogue scale (VAS) before GP (Figure 2).

It was treated by combination of antibiotics, prescribed by two subsequent antibiogram, and prostacyclin infusion (iloprost, $0.5 \mathrm{ng} / \mathrm{kg} / \mathrm{min}$; Italfarmaco S.p.a., Milano, Italy) on an outpatient basis. To achieve the goal of outpatient management we adopted an elastomeric pump which was daily loaded with iloprost for five days a week, for three weeks duration. ${ }^{17,18}$ Despite this treatment coupled with standard wound 
care, necrosis and ulceration did not improve; in addition, pain was not supported without major drugs. We added GP at home, two cycles a day for duration of 30 min per cycle. We also added standard wound care twice a week and compression with below knee elastic stocking exerting 20-30 $\mathrm{mmHg}$ at the ankle (Flebysan S.r.l., Rovigo, Italy). This resulted in limb salvage out of loss of three distal phalanges of the toes, total control of pain without any drug (Table 1).

\section{Case \#2}

Patient B is a 77-year old female affected by MLU of the lateral and posterior aspect of the left leg with rest pain no more responding to major drugs. MLU was due to occlusion of iliac femoral by-pass performed three years before coupled with post thrombotic syndrome of the left leg, in consequence of a venous thrombosis occurred 15 years before (Table 1). She was treated by home-based GP, two cycles a day for duration of 30 min per cycle. We also added wound care and inelastic bandaging weekly changed. Finally, she was treated by combination of antibiotics, prescribed by two subsequent antibiogram, and prostacyclin infusion on outpatient basis thanks to an elastomeric pump which was daily substituted for five days a week, for three weeks duration. ${ }^{17,18}$ This strategy resulted in significant reduction of pain, reduction of analgesic use and total limb salvage (Figure 3).

\section{Case \#3}

Patient $\mathrm{C}$ is a 67 -year old male affected by MLU due to superficial venous insufficiency of the right great saphenous vein (GSV), combined with PAD II stage with a vast necrotic ulceration of the posterior and medial aspect of the leg (Table 1). He was treated by echoguided foam sclerotherapy of the GSV according to Tessari's method, in order to suppress the superficial reflux..$^{19,20}$

In addiction, we performed surgical debridement followed by inelastic compression bandaging and antibiotics based on three subsequent antibiograms. Moreover, we used prostacyclin infusion on outpatient basis thanks to an elastomeric pump, which was daily loaded with Iloprost for five days a week, for three weeks duration. ${ }^{17,18}$ Finally, he was treated by home-based GP, two cycles a day for duration of 30 min per cycle. This resulted in wound healing and total pain control with suspension of analgesic drugs (Figure 4).

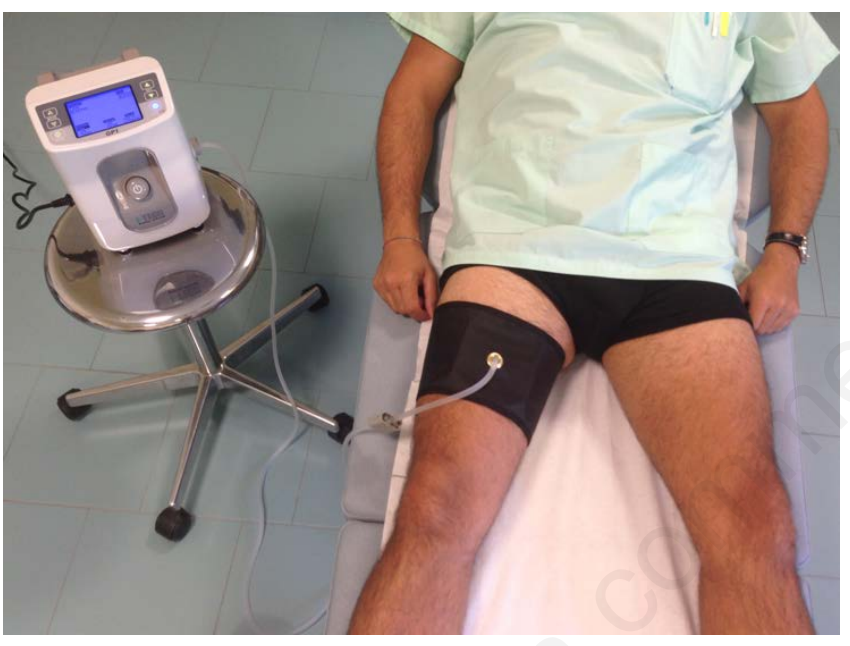

Figure 1. Gradient pump treatment and proper placement of the cuff.

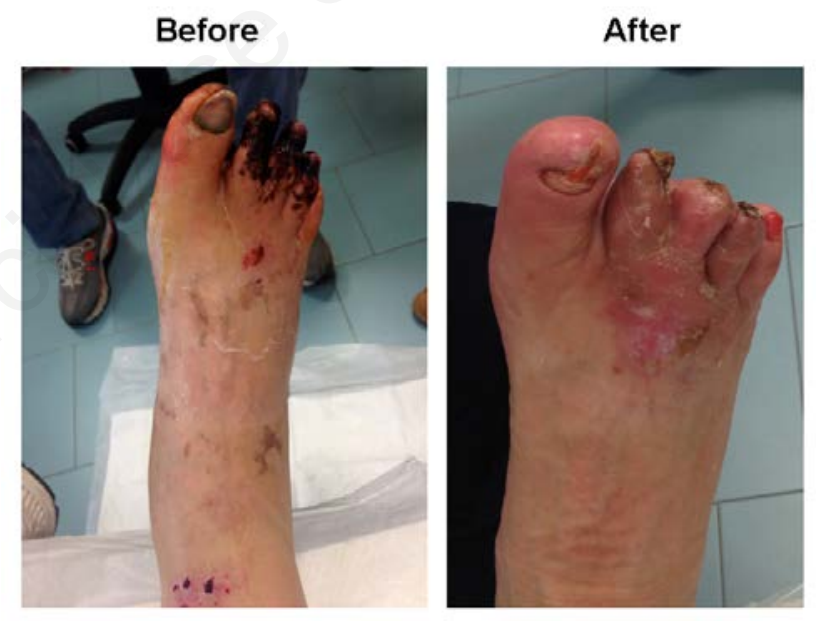

Figure 2. Clinical case of patient A before and after treatment of gradient pump.

Table 1. Reported cases: demographics, clinics and outcome measure.

\begin{tabular}{|c|c|c|c|c|c|c|c|c|c|c|}
\hline Patient & $\begin{array}{l}\text { it Age; } \\
\text { gender }\end{array}$ & $\begin{array}{c}\text { PAD stage; } \\
\text { ABI }\end{array}$ & $\begin{array}{l}\text { Venous } \\
\text { diagnosis }\end{array}$ & $\begin{array}{l}\text { Venous } \\
\text { treatment }\end{array}$ & $\begin{array}{l}\text { Arterial } \\
\text { treatment }\end{array}$ & $\begin{array}{c}\text { Compliance to } \\
\text { GP }\end{array}$ & $\begin{array}{l}\text { VAS } \\
\text { before }\end{array}$ & $\begin{array}{l}\text { VAS } \\
\text { after }\end{array}$ & Outcome & $\begin{array}{l}\text { Drugs before; } \\
\text { drugs after }\end{array}$ \\
\hline A & 57 ; male & Il stage; 0.7 & PTS & $\begin{array}{l}\text { Elastic } \\
\text { stocking }\end{array}$ & Iloprost i.v. & Yes & 7 & 2 & $\begin{array}{c}\text { Healing and } \\
\text { phalanges amputation }\end{array}$ & NSAID; suspended \\
\hline B & 77; female & II stage; 0.6 & PTS & $\begin{array}{c}\text { Inelastic } \\
\text { bandaging }\end{array}$ & Iloprost i.v. & Yes & 10 & 4 & Healing & Opioids; NSAID \\
\hline $\mathrm{C}$ & 67 ; male & II stage; 0.6 & $\begin{array}{c}\text { Primary } \\
\text { superficial } \\
\text { CVI }\end{array}$ & $\begin{array}{c}\text { Foam } \\
\text { sclerotherapy } \\
\text { and inelastic } \\
\text { bandaging }\end{array}$ & Iloprost i.v. & Yes & 8 & 2 & Healing & NSAID; suspended \\
\hline D & 36 ; male & II stage; 0.6 & $\begin{array}{c}\text { Primary } \\
\text { superficial } \\
\text { CVI } \\
\text { and thrombosis }\end{array}$ & $\begin{array}{l}\text { Inelastic } \\
\text { bandaging } \\
\text { s }\end{array}$ & $\begin{array}{c}\text { None, } \\
\text { allergic } \\
\text { to endoprost }\end{array}$ & Yes & 9 & 5 & Healing & NSAID; suspended \\
\hline
\end{tabular}

PAD, peripheral arterial disease; ABI, ankle brachial index; GP, gradient pump; VAS, visual analogue scale; PTS, post-thrombotic syndrome; i.v., intravenously; NSAID, non-steroidal anti-inflammatory drug; CVI, chronic venous insufficiency. 


\section{Case \#4}

Patient D is a 36 -year old male with primary chronic venous insufficiency clinical class 2 of the Clinical-Etiology-Anatomy-Pathophysiology (CEAP) classification, operated on in emergency for critical limb ischemia in consequence of idiopathic thrombosis of the right femoro-politeal segment complicated by distal micro embolism. An in situ saphenous vein femoro-posterior tibial by pass has been performed. However, despite the patency of the by pass, the patient developed small and painful ulceration the second and third finger of the right foot due to distal embolization. He was allergic to endoprost and the ulcers were treated exclusively by GP. In four days the VAS passes from 9 to 5 (Table 1). He was able to stand and walk some steps. After hospital discharge he continued at home the GP treatment, 35 min three times a day. Ulcer healed in two weeks and pain disappeared in one week.

\section{Discussion}

We herein report four challenging cases of MLU. The main finding of our cases report is the possibility of successful treatment of the painful conditions on outpatient bases. This was possible thank to three common points in the strategy used: i) home-based GP treatment: it has been demonstrated in a previous study how GP improves distal perfusion. ${ }^{2}$ In the present study we demonstrate the feasibility of GP treatment at home with full compliance of patients and/or family trained in hospital. The device is very simple to be used and two cycles a day demonstrated to be very well tolerated, especially because the compression is at the thigh (Figure 1) so very distant from the painful lesion. IPC equipment usually pump at the level of foot and leg, so increasing pressure against the surface of the ulcer, limiting their
Before

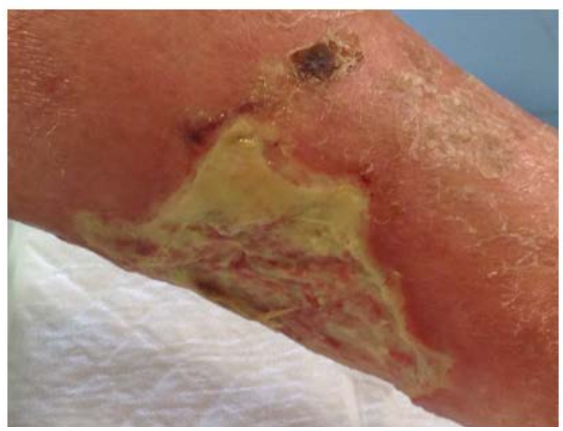

After

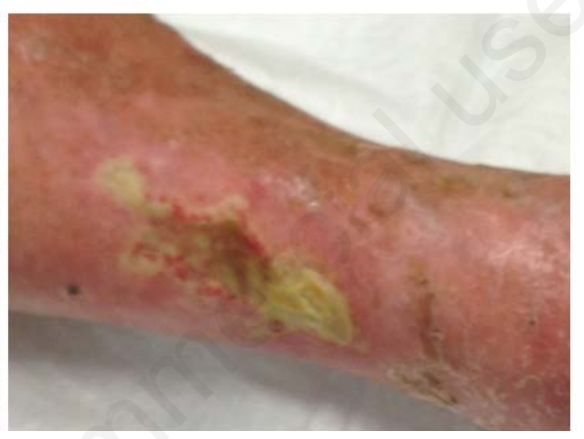

Figure 3. Clinical case of patient $B$ before and after treatment of gradient pump.

Before

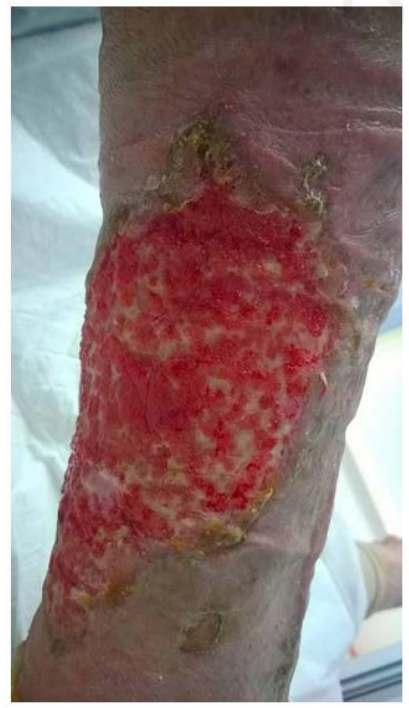

After

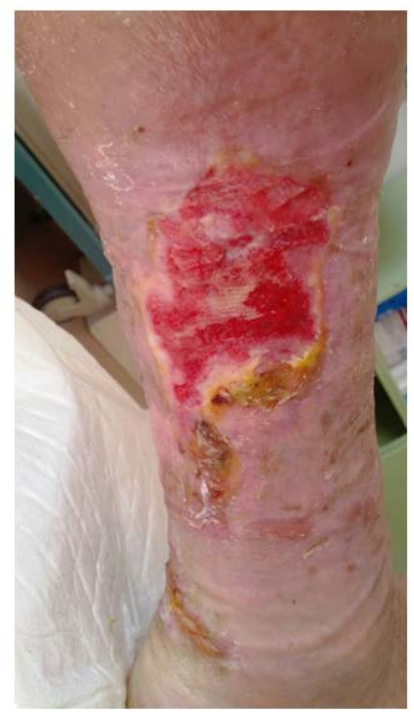

Figure 4. Clinical case of patient $\mathrm{C}$ before and after treatment of gradient pump.

use for loss of tolerance on behalf of the patients. To the contrary, our experience demonstrates a significant reduction of pain measured by VAS (Table 1); ii) elastomeric pump infusion of prostacyclin: the elastomeric pump permits to continue the drug infusion along the day, after loading and controlling the patients each morning on ambulatorial bases. Iloprost was injected through a peripheral venous catheter at $0.5 \mathrm{ng} / \mathrm{min}$ for $24 \mathrm{~h}$, five days a week. On Saturday and Sunday drug was stopped, because the service in hospital was closed. Cardio-vascular parameter was checked in the first 2-3 $\mathrm{h}$ from beginning, while the patient was in hospital. This is an innovative management of prostacyclin administration which permits to reduce the cost of hospitalization; iii) wound care, compression and eventual venous treatment: wound care, including debridement was achieved on ambulatorial bases, according to standard care. Our study concurs to demonstrate that inelastic bandage can be used in MLU without increasing pain. Finally, the case where we stopped reflux by mean of echo-guided foam sclerotherapy, again confirmed how this treatment is effective and feasible on ambulatorial bases. ${ }^{19,20}$

The contribution of GP to the successful clinical outcome of the reported cases cannot be clarified by the present observational and preliminary report. However, patient D with endoprost intolerance, suggests us that GP can be used also in the absence of adjuvant pharmacological treatment

The major limitation of our study is that actually GP is still a prototype. However, the course for CE license is in progress and it will be available shortly on the market. A second short-coming is represented by the number of patients, but this was a pilot study which paves the way to further multicentric evaluations of GP in MLU, when the devices will be available in other centers.

\section{Conclusions}

GP represents an interesting opportunity in the management of MLU, improving leg perfusion, reducing pain, and for the high tolerance and compliance of the patients, as well.

GP versatility permits a home treatment with considerable lowering of cost.

\section{References}

1. Krishna SM, Moxon JV, Golledge J. A Review of the pathophysiology and potential biomarkers for peripheral artery disease. Int J Mol Sci 2015;18:11294-322. 
2. Manfredini F, Malagoni AM, Felisatti M, et al. Acute oxygenation changes on ischemic foot of a novel intermittent pneumatic compression device and of an existing sequential device in severe peripheral arterial disease. BMC Cardiovasc Disord 2014:31:14-40.

3. Tessari M, Malagoni AM, Vannini ME, et al. A novel device for non-invasive cerebral perfusion assessment. Veins and Lymphatics 2015;4:4650.

4. Anderson I. Mixed aetiology: complexity and comorbidity in leg ulceration. Br J Nurs 2008;17:17-8.

5. Crawford F, Anandan C, Chappell FM, et al. Protocol for a systematic review and individual patient data meta-analysis of prognostic factors of foot ulceration in people with diabetes: the international research collaboration for the prediction of diabetic foot ulcerations (PODUS). BMC Med Res Methodol 2013;13:22.

6. Stevens J. Diagnosis, assessment and management of mixed aetiology ulcers using reduced compression. J Wound Care 2004;13:339-43.

7. Jochmann W, Mostbeck A, Partsch H. Post occlusive reactive hyperemia and postural vasoconstriction in different kinds of leg ulcers-investigations with laser Doppler. Vasa 1993;22:306-15.

8. Rümenapf G, Geiger S, Schneider B, et al. Readmissions of patients with diabetes mellitus and foot ulcers after infrapopliteal bypass surgery - attacking the problem by an integrated case management model. Vasa 2013;42:56-67.

9. Mosti G. Compression in leg ulcer treatment: inelastic compression. Phlebology 2014;29:146-52.

10. Mosti G. Compression in mixed ulcers: venous side. Phlebology 2014;29:13-7.

11. Gonzàlez-Consuegra RV, Verdù J. Quality of life in people with venous leg ulcers: an integrative review. J Adv Nurs 2011;67: 926-44.

12. Herber OR, Schnepp W, Rieger MA. A systematic review on the impact of leg ulceration on patients' quality of life. Health Qual Life Outcomes 2007;5:44.

13. Xie X, McGregor M, Dendukuri N. The clinical effectiveness of negative pressure wound therapy: a systematic review. J Wound Care 2010;19:490-5.

14. Vig S, Dowsett C, Berg L, et al. Evidencebased recommendations for the use of negative pressure wound therapy in chronic wounds: steps towards an international consensus. J Tissue Viability 2011;20:1-18.

15. Bovill E, Banwell PE, Teot L, et al. Topical negative pressure wound therapy: a review of its role and guidelines for its use in the management of acute wounds. Int Wound J 2008;5:511-29.

16. Lawall $\mathrm{H}$. Treatment of chronic wounds. Vasa 2012;41:396-409.

17. Lambert MA, Belch JJ. Medical management of critical limb ischaemia: where do we stand today? J Intern Med 2013;274: 295-307.

18. Piaggesi A, Vallini V, Iacopi E, et al. Iloprost in the management of peripheral arterial disease in patients with diabetes mellitus. Minerva Cardioangiol 2011;59:101-8.

19. Tessari L. [Nouvelle technique d'obtention de la sclero-mousse.] Phlebologie 2000;53:129. [Article in French]

20. Tessari L, Izzo M, Cavezzi A, et al. Timing and modality of the sclerosing agents binding to the human proteins: laboratory analysis and clinical evidences. Veins and Lymphatics 2014;3:3275. 